\title{
LAS MUJERES MAYORES, LOS CUIDADOS Y LOS CLUBES: NEGOCIACIONES INTERSUBJETIVAS DE UNA ETNOGRAFÍA FEMINISTA
}

\begin{abstract}
Herminia Gonzálvez ToRralbo Universidad Central de Chile. Instituto de Investigación y Postgrado, Facultad de Derecho y Humanidades herminiagonzalvez@gmail.com
\end{abstract}

MENARA LUBE GUIZARDI Universidad de Tarapacá (Chile). Consejo Nacional de Investigaciones Científicas y Técnica de Argentina. Instituto de Altos Estudios Sociales

(IDAES-UNSAM) menaraguizardi@yahoo.com.br

REsumen: El artículo discute los procesos de intersubjetividad de una etnografía acerca de las prácticas femeninas de cuidado comunitario que se expresan en los clubes de personas mayores en Santiago de Chile. En un primer momento, situaremos los inicios de nuestro interés por el envejecimiento femenino vinculándolo a los hallazgos de un proyecto de investigación anterior $y$, simultáneamente, por inesperados sucesos personales. En segundo lugar, dilucidaremos cómo hemos diseñado un nuevo proyecto proponiendo una etnografía feminista que diera protagonismo a las mujeres mayores en su rol como constructoras de redes femeninas de cuidado comunitario. En el tercer momento, entraremos a la trastienda de la investigación reflexionando sobre la negociación de las distinciones que hemos desarrollado con estas mujeres en sus clubes. Concluimos invitando a una reflexión sobre el lugar de la intersubjetividad en procesos etnográficos en los cuales la experiencia comunitaria está interpelada por la reciprocidad de los cuidados femeninos. 
Palabras ClaVE: mujeres mayores; cuidados; etnografía feminista; intersubjetividad.

AвstRACT: The article discusses the intersubjectivity processes in an ethnographical study into the feminine practices of community care that occur in the clubs of older people in Santiago de Chile. First, we describe our interest in female aging and its connections to the findings of a previous research project and, simultaneously, our interest in unexpected personal events. Second, we describe how we have designed a new project proposing a feminist ethnography that gives prominence to older women in their role as the builders of feminine networks of community care. Third, we enter the back room of the investigation by reflecting on the negotiation of the distinctions that we have developed with these women in their clubs. We conclude by reflecting on the place of intersubjectivity in ethnographic processes in which the community experience is challenged by the reciprocity of female care.

KEYWORDS: older women; care; feminist ethnography; intersubjectivity. 


\section{Introducción}

En este artículo, les invitamos a conocer la «trastienda» de una investigación antropológica sobre los cuidados comunitarios de mujeres mayores en Santiago de Chile. Nuestro propósito principal es compartir específicamente las etapas y reflexiones metodológicas que nutrieron nuestra «entrada al campo» en un proceso investigativo marcado por nuestro compromiso (y deseo) de llevar a cabo una etnografía feminista. A contracorriente de lo que usualmente se espera de las investigadoras en un artículo etnográfico, no ahondaremos en los resultados de la investigación, sino que nos centraremos en aquellos momentos cruciales de nuestro proceso investigativo que nos permitieron alcanzar dichos resultados. Este ejercicio nos permitirá entregar la información que, usualmente, permanece en los bastidores de las investigaciones, aquella relacionada con el posicionamiento epistemológico feminista desde el que se sitúa nuestro quehacer durante el trabajo de campo. Una suerte de material que los antropólogos suelen tratar como «clasificado», pero cuya exposición puede contribuir fehacientemente a la superación de las encrucijadas con las cuales nos encontramos a la hora de poner en práctica la etnografía feminista.

Partiremos nuestra exposición situando los inicios de nuestro interés por la vejez femenina, remontándonos a una primera investigación antropológica realizada entre 2012 y 2015 , centrada en el estudio del trabajo que realizan las mujeres -en sus diferentes edades - para mantener el sentimiento de pertenencia entre quienes ellas consideran como parte de su familia. Esta investigación situó el tránsito entre nuestra preocupación por el trabajo de reproducción social que realizan las mujeres en general y el trabajo de sostenimiento de la vida que realizan las mujeres mayores en particular. Pero, sinceraremos que, además de estos elementos empíricos, nuestra atención fue llevada al tema debido a experiencias personales, lo que ha provocado que la investigación tuviera, desde su inicio, un carácter intersubjetivo.

Nuestra siguiente parada aborda una segunda investigación, construida a partir de los interrogantes sembrados por los resultados de la anterior y desarrollada entre 2016 y 2019. En este nuevo proyecto, nos 
propusimos comprender el proceso de envejecer de las mujeres. A partir de una detenida revisión sobre cómo las ciencias sociales han abordado el envejecimiento femenino, llegamos a nuestra propuesta de cómo abordar etnográficamente este tema, buscando comparar las experiencias femeninas ubicadas en diferentes sectores socioeconómicos de la capital chilena. Esto nos llevó a realizar nuestra etnografía en los clubes gestionados por mujeres mayores que, conforme observamos, constituyen respuestas colectivas a sus demandas del cuidado.

Nuestro tercer eje analítico emerge a la luz del inicio de nuestro trabajo de campo, orientado por los propósitos y compromisos de la etnografía feminista. En el marco de este debate, explicitaremos, por un lado, nuestra particular forma de aproximarnos a dichos espacios de la mano de la participación observante, y, por otro lado, nuestra manera de construir las relaciones con las mujeres mayores. Detallaremos, entonces, cómo la investigación nos empujó a procesos de negociación de las distinciones sociales (las nuestras y las de las mujeres), problematizando no solo nuestra posición, sino también nuestra relación, de ida y vuelta, con ellas:

Al dejar de considerarlas como informantes para, a partir de la teoría antropológica feminista, considerarlas como creadoras culturales $y$, al mismo tiempo, identificar, analizar e interpretar las orientaciones, contenidos y sesgos de género que las colocan a ellas, a los varones y a otras categorías sociales genéricas en posiciones diferenciadas que, en la mayoría de los casos, atañen a las desigualdades entre unas y otras (Castañeda, 2012: 221).

Finalizamos con reflexiones respecto de cómo redimensionar el lugar de los cuidados comunitarios como elemento articulador del cuidado de sí y de otros y otras. Abordando el carácter recíproco de las lógicas del cuidado femenino de los clubes etnografiados, retomaremos el papel fundamental que la intersubjetividad tiene en la praxis de la etnografía feminista. Consideramos que este ejercicio analítico se vuelve fundamental en estos momentos de múltiples crisis (económica, social, de cuidados) enfrentados globalmente y que demandan de los investigadores redoblar nuestro compromiso con la empatía. Demanda, además, asegurar la autovigilancia en lo que concierne a nuestra actuación profesional, centra- 
lizando el respeto por el derecho al cuidado de las personas cuyas vidas y experiencias investigamos.

\section{Adentrando al tema}

El inicio de nuestro interés por el cuidado comunitario que realizan las mujeres mayores en Santiago de Chile se remonta a una investigación realizada entre 2012 y $2015^{1}$. Este estudio, de corte antropológico y feminista, se centró en mostrar el trabajo diferenciado que hacen hombres y mujeres para mantener el sentimiento de pertenencia entre aquellas personas que consideran de su familia, lo que algunas autoras denominan «trabajo de parentesco» (Di Leornado, 1987; Gonzálvez y Acosta, 2015; Gonzálvez, 2016; 2017).

A través de la realización de entrevistas en profundidad ${ }^{2}$, indagamos por los contenidos de ese trabajo de parentesco, es decir, por aquellas celebraciones y rituales que se expresan al interior del grupo doméstico y que incluyen prácticas tan diversas como hacer visitas, enviar cartas, realizar llamadas de teléfono, hacerse regalos, organizar las vacaciones o preparar los encuentros por Navidad, por mencionar solo algunas (Di Leonardo, 1987: 442). Así, pudimos visibilizar aquellas prácticas sociales asociadas a los cuidados que producen y reproducen relaciones de parentesco e identidad colectiva, pero que no son consideradas como valiosas por la naturalización que comporta su asociación con lo femenino y lo doméstico (Gregorio y Gonzálvez, 2012).

Si bien esta investigación produjo diferentes resultados, lo que más llamó nuestra atención fue, por un lado, que un número no menor de personas entrevistadas, quienes realizaban gran parte de este trabajo de

I El estudio referido fue financiado por el Fondo Nacional de Desarrollo Científico y Tecnológico (FONDECYT) de la entonces Comisión Nacional de Investigación Científica y Tecnológica (actual Agencia Nacional de Investigación y Desarrollo de Chile), a través del Proyecto de Iniciación n. ${ }^{\circ}$ 11121245: «Las familias en Chile: El trabajo de parentesco y la generación de constelaciones familiares». El equipo de investigación estuvo conformado por Herminia Gonzálvez Torralbo (investigadora responsable), Francisca Ortiz y Sofia Larrazabal (asistentes de investigación).

2 Se realizaron 33 entrevistas durante la primera etapa del trabajo de campo (octubre 2012-septiembre 2013) y 26 entrevistas en la segunda (octubre 2013-septiembre 2014). Pero con la particularidad de que, adoptándose una metodología de carácter longitudinal, se repetían estas entrevistas a las mismas personas en los tres años de duración del proyecto. 
mantenimiento de los vínculos, eran mujeres. Además, muchas de ellas eran mayores y vivían solas. Por otro lado, que en sus discursos asociados a cómo mantenían estos vínculos familiares se describía una multiplicidad de prácticas realizadas no solamente con familiares consanguíneos, sino $\mathrm{y}$, sobre todo, con los amigos y las amigas, con los vecinos y las vecinas. Así, los resultados de la investigación arrojaron que las mujeres mayores eran protagonistas de los cambios sociodemográficos que estaba experimentando la sociedad chilena, debido al alargamiento de la esperanza de vida y el consecuente envejecimiento de la población, y que afrontaban esta realidad a través de una activa participación comunitaria.

Estas constataciones de orden antropológico-empírico fueron interpeladas por procesos personales: nuestro ímpeto por sentir y pensar el envejecimiento femenino fue parte de un proceso que desbordó fronteras geográficas, entrecruzando y entretejiendo, simultáneamente, dimensiones personales y profesionales. Esto ha dotado todo cuanto hemos desarrollado posteriormente de una dimensión centralmente intersubjetiva que terminó caracterizando no solamente la propuesta de investigación que se creó, sino también nuestra manera de vincularnos con las mujeres mayores. Por lo general, en las ciencias sociales, las investigadoras evitamos mencionar estas razones personales. Rompiendo este protocolo -que nos deshumaniza, a la par que elude la dimensión intersubjetiva crítica de nuestras etnografías-, sinceraremos aquí nuestras experiencias que nos empujaron a indagar sobre cómo las mujeres afrontan los cuidados en la vejez.

Para Gonzálvez, el tema se volvió algo personal unos meses después de la finalización del proyecto anterior, a mediados de 2016. En este momento, ella se enfermó y tuvo que operarse de urgencia: fue un año de diversos desafíos de salud vinculados a este episodio. Como es migrante en Chile, vive lejos de sus redes femeninas familiares. Mientras se recuperaba, no pudo dejar de pensar en las mujeres mayores de la investigación concluida; no pudo dejar de pensar en la transcendencia de esta función femenina del cuidado desempeñada por ellas y su importancia para que mujeres que - como ella misma - necesitan descansar en el apoyo de las mayores para sostener sus funciones productivas. Ella lo comprendió, 
entre otras cosas, porque debió usar toda su capacidad argumentativa para evitar que su madre viajara los casi once mil kilómetros que separan su ciudad natal, Elche (en el Estado español), de Santiago de Chile, para acompañarla con su incondicional trabajo de cuidado. En este momento, todo cuanto Gonzálvez había escuchado y comprendido en la etnografía se convirtió en una experiencia corporal, encarnada, afectiva.

Para Guizardi, el tema se volvió muy personal desde finales de 2015, cuando su madre se enfermó de cáncer. Con sus dos hermanas, tuvo que hacerse cargo de cuidarla, asistiendo a su padre, que, pese a sus grandes esfuerzos y genuinas intenciones, pasó toda una vida sin cuidar y carecía de herramientas para hacerlo. La tarea fue de las más difíciles. Las tres hermanas tuvieron que hacer malabarismos para atender simultáneamente a sus actividades productivas. Dos no vivían en Brasil, donde residían los progenitores, debiendo convertirse en cuidadoras multisituadas, viajando varios kilómetros cada mes. Pero, además, tuvieron que enfrentar la resistencia materna. Primera generación universitaria de la familia, su madre rechazó tajantemente que el cuidado (de personas y doméstico) debiera ser una obligación femenina. Se propuso educar a las hijas para no asumir al cuidado como un destino obligado: consecuentemente, se negaba a ser cuidada por ellas en la enfermedad. Tras la muerte de su madre, Guizardi contó estas experiencias a Gonzálvez, quien la terminó invitando a integrar el proyecto que origina este artículo.

Entre las dos, llegamos a una conclusión transcendente: como migrantes que debemos hacernos cargo de envejecer lejos de nuestras redes femeninas, nos toca asumir formas comunitarias de cuidado que construyen redes con amigas y colegas. En gran medida, nuestra vinculación como antropólogas había nacido de este trabajo del parentesco: estudiamos el doctorado en una misma universidad de Chile, compartimos redes con nuestros respectivos orientadores. Pero solo nos volvimos muy cercanas cuando ambas migramos a Chile en pleno contexto de la crisis económica que impactó Europa a partir de 2008. Así, nos sorprendimos con la constatación de que, como las mujeres que Gonzálvez había investigado, nos tocaba envejecer y cuidarnos entre nosotras. 
Estas vivencias, constataciones y experiencias nos empujaron a indagar antropológicamente sobre las mujeres, los cuidados y el envejecimiento en un momento en que el contexto chileno también parecía empujarnos al tema. Los datos sociodemográficos apuntaban a la aceleración del proceso de envejecimiento en Chile. El discurso político estaba en pleno cambio: de enunciados reconocedores de que el país estaba envejeciendo a otros que aludían al envejecimiento de la población como un hecho. Chile empezaba a organizar social y políticamente los cuidados de la población mayor a partir del compromiso anunciado en la cuenta pública del año 2015 por la entonces presidenta de la república, Michelle Bachelet, de la creación del Subsistema Nacional de Apoyos y Cuidados (SNAC)3. Este sistema comprendería un conjunto de acciones públicas y privadas relativas a entregar apoyo y cuidados para personas en situación de dependencia.

Fue el conjunto de nuestras constataciones personales, aquellas derivadas de los resultados de investigación que había dirigido Gonzálvez, unido a las que observábamos cotidianamente en el contexto social y político, lo que nos impulsó, definitivamente, a abrazar como «nuestro» el tema del envejecimiento femenino.

Decididas a convertir nuestras inquietudes en etnografía, postulamos a un proyecto de investigación que tratara únicamente sobre mujeres mayores. Nos interesaba saber cómo se envejecía en Chile siendo mujer. Debido a los resultados del proyecto anterior, sabíamos que las prácticas comunitarias de cuidado realizadas por las mujeres mayores tenían mucha centralidad para ellas y su entorno social ${ }^{4}$. Comprendimos, entonces, que era necesario mirar al territorio e indagar cómo las mujeres desplegaban en sus comunidades prácticas que les permitían asistirse mutuamente en el proceso de envejecer.

3 Posteriormente llamado Sistema Nacional de Apoyo y Cuidados, y coloquialmente Chile Cuida, aunque no son equivalentes.

4 Para mayor conocimiento y profundización sobre dichos resultados, véanse Gonzálvez y Larrazábal (2019), Gonzálvez et alii (2018), Ortiz y Gonzálvez (2017), y Gonzálvez (2016). 


\section{Los cuidados comunitarios y los clubes de personas mayores}

Nuestra postulación resultó exitosa: logramos adjudicarnos un proyecto de investigación de tres años (2016-2019) para profundizar etnográficamente en la experiencia del envejecimiento femenino en Santiago de Chile ${ }^{5}$. En el marco de dicho proyecto, nos propusimos analizar cómo la organización social, moral y política de los cuidados comunitarios impactaba los procesos de envejecimiento de las mujeres de tres comunas de la Región Metropolitana de Santiago: Independencia, Santiago Centro y Providencia ${ }^{6}$. Seleccionamos estas tres comunas, y no otras, por dos características sociodemográficas que hacen de ellas espacios condensadores de las problemáticas femeninas que buscamos comprender. Por un lado, están entre las tres más feminizadas y también entre las más envejecidas de la Región ${ }^{7}$. Es decir, en ellas, se observa una prevalencia de las mujeres entre la población adulta mayor. Por otro lado, presentan perfiles de estratificación socioeconómica diversificados: bajo (en el caso de Independencia), mediano (Santiago) y alto (Providencia), permitiéndonos comparar el envejecimiento femenino a partir de las desigualdades y diferencias que las diversas condiciones socioeconómicas articulan. Esto implicaba comprender, por un lado, el desajuste entre las necesidades de cuidados de las personas mayores y su inequitativa respuesta por parte de la familia, la comunidad, el mercado y el Estado. Y, por otro lado, las valoraciones sociales y morales del cuidado operantes en la sociedad (normativas, creencias).

\footnotetext{
5 Dicha investigación fue financiada por CONICYT (actualmente, ANID), a través del proyecto Fondecyt Regular n. ${ }^{\circ} 1160683$ : «Ser mujer mayor en Santiago de Chile: Organización social de los cuidados, feminización del envejecimiento y desigualdades acumuladas», dirigido por Herminia Gonzálvez Torralbo.

6 Chile se encuentra dividido en dieciséis unidades territoriales llamadas «regiones», que están a cargo de un intendente designado por el presidente de la República. Las regiones se subdividen, a su vez, en provincias (a cargo de un gobernador, igualmente designado por el presidente), y estas, en comunas (dirigidas por un alcalde electo por votación popular). La región donde se encuentra la capital del país, Santiago de Chile, es denominada Región Metropolitana, y está subdivida en seis provincias y 52 comunas.

7 En la Región Metropolitana existen otras comunas también envejecidas, como Nuñoa, La Reina o Vitacura, por mencionar algunas. Pero las tres que elegimos se diferencian de las demás porque están fuertemente feminizadas en términos de envejecimiento.
} 
Paralelamente, nuestra detenida revisión de los estudios sobre los contextos nacionales chilenos y de otros países latinoamericanos nos ayudó a recopilar varias constataciones empíricas que dieron cuenta de la complejidad del fenómeno que nos proponíamos abordar. Primero, los estudios demográficos y gerontológicos demostraban que la vejez es una etapa del ciclo de vida que es cada vez más duradera, y desde la que se prolongan, retoman o continúan responsabilidades más allá de las esperadas: no solo en cuanto al lugar que ocupan las personas mayores como hombres y mujeres, padres, madres, o abuelos/as, sino también por la continuidad de sus responsabilidades en la provisión económica del hogar, en estrecha relación con el cuidado de ellos mismos y/o de sus familiares.

Segundo, que el proceso de envejecer es desigual en cuanto al género-parentesco y la clase social —entre otras categorías de diferenciación social-, lo que significa que no es lo mismo envejecer siendo hombre o mujer (De Beauvoir, (1983[1970]; Freixas, 1997; Huenchuan, 2014), abuelo o abuela, con recursos o sin recursos, trabajando o sin trabajar remuneradamente. A su vez, estas constataciones nos remitían a nuestra postura política situada desde la antropología feminista ${ }^{8}$.

Tercero, que los cuidados son una necesidad diaria de todas las personas, que, si bien adquieren formas diversas en función del ciclo vital y del contexto histórico y cultural en el que se ellas encuentran (Precarias a la Deriva, 2004: 210), ganan un gran protagonismo durante la vejez.

Frente a esto, nos llamó la atención, por un lado, que las mujeres mayores hayan sido, hasta avanzado el siglo xxi, sujetos poco protagónicos en las ciencias sociales en general (Gibson, 1996; Freixas, 1997; Huenchuan, 2014), y en los estudios desarrollados en Chile, en particular (Gonzálvez y Guizardi, 2020). Por suerte, desde la primera década del presente siglo, investigadores e investigadoras vienen contribuyendo a visibilizar este tema en el país, corrigiendo, así, una asimetría histórica en la producción académica (Barros et alii, 2014; Forttes, 2016; Gallardo et alii, 2015; 2017;

8 Aplicar la perspectiva feminista desde la antropología social y cultural (Del Valle, 2010; Gregorio, 2011) implicó detenerse en el examen de las desigualdades sociales de género a partir del análisis de la vivencia de la vejez de las mujeres mayores. 
Herrera, 2011; Mazzucchelli y Arévalo, 2019; Mazzucchelli, 2019; Osorio, 2006; 2007).

Por otro lado, nos impresionó observar que el cuidado, como una categoría de análisis social y política, hubiera emergido solo recientemente como un problema de investigación concerniente a los científicos/as sociales, más allá del hecho que desde las investigaciones feministas se lleve reivindicando su importancia desde hace más de treinta años (Hanlon, 2012; Gonzálvez, 2016). También, que la familia se constituya como aquel lugar desde donde se ha producido mayor información respecto de la entrega y la recepción de cuidados a diferencia de lo que sucede en relación con la comunidad, influyendo en la imprecisión que existe respecto de lo que se entiende por cuidados comunitarios desde las ciencias sociales (Gonzálvez et alii, 2019: 140). Por último, que el estudio de las múltiples desigualdades de género-parentesco y clase social (Gonzálvez, 2010; 2011; Comas d'Argemir y Soronellas, 2019), entre muchas otras, asociadas al proceso de envejecer todavía hoy sea algo incipiente, más allá de la rotundidad de los datos que nos muestran que el envejecimiento en Chile se encuentra feminizado, y que es profundamente desigual entre diferentes sectores socioeconómicos (Osorio, 2007; CASEN, 2013; 2015a; 2017; INE, 2017).

Tras revisar dichos estudios, y poner atención al contexto sociodemográfico, optamos por incorporar en nuestro análisis la triada analítica entre desigualdad(es) sociales(es), envejecimiento y cuidados para, desde allí, indagar sobre su relación con el «trabajo de cuidado», y más específicamente, con el cuidado comunitario. Consecuentemente, y en el marco de dicha investigación, nos propusimos conocer cómo operan el Estado, el mercado, la familia y la comunidad — que juntos constituyen el «diamante del cuidado» (Rodríguez, 2015) - en las tres comunas de la Región Metropolitana de Chile seleccionadas.

Nuestro acercamiento a las experiencias femeninas del envejecimiento en cada comuna se dio a través de un recorte cualitativo muy particular. Entre 2016 y 2018, participamos de los clubes gestionados por mujeres mayores de 60 años. Estas organizaciones son conocidas popularmente en Chile como «clubes de adultos mayores». Son espacios fundamenta- 
les para la sociabilidad de vecinos y vecinas en diversas comunas de todo país. Muchos de estos clubes fueron creados a partir de una organización comunitaria popular de base conocida en la segunda mitad del siglo xx en Chile como «clubes de madres». Dichas organizaciones tuvieron una historia y una trayectoria políticas complejas, acoplándose diferentes orientaciones partidarias dependiendo de los Gobiernos de turno9. Pero, más allá de estas transformaciones políticas, persistieron como organizaciones sociales con notorio protagonismo femenino. Tanto los clubes de madres como los de adultos mayores son frecuentados y gestionados por una mayoría femenina hasta la actualidad, como veremos a continuación. Las mujeres se autoconvocan en estos espacios a partir de intereses compartidos, retomando una historia popular de configuración de redes comunitarias de cuidado. Nos hicimos asiduas de tres clubes en particular (uno por comuna), en cuyas actividades - talleres artísticos, paseos turísticos, eventos de recaudación de fondos, comidas, meriendas- pudimos intimar con las mujeres que fueron las protagonistas de la investigación que desarrollamos. En el apartado que sigue profundizaremos en esta experiencia intersubjetiva.

\section{Nuestra aproximación}

Buscando acercarnos a las experiencias de los clubes de personas mayores, nuestra estrategia inicial fue la de identificar aquellos talleres ofertados por las municipalidades de las tres comunas seleccionadas y gestionados por dichos clubes ${ }^{10}$. Dar cumplimiento a este propósito fue un gran desafío, debido, por un lado, a que las Administraciones municipales no tenían disponible esta información en la web y, por otro lado, a que la información entregada, en algunos casos, se encontraba desactualizada.

Para conseguirla, acudimos presencialmente a las oficinas encargadas de la política hacia la población mayor y preguntamos directamente por los contenidos de los talleres ofertados y sus horarios. En la medida en que comenzamos a conocer las actividades ofertadas para las personas

9 Para más detalles sobre la historia de estas organizaciones, véase Gonzálvez et alii (2019). 10 Según la ley vigente en Chile, la administración de las comunas queda a cargo de un organismo del poder ejecutivo denominado «municipalidad». 
mayores, nos dimos cuenta de que los talleres constituían un espacio fundamental de socialización de las personas mayores en sus territorios de vivienda. Los funcionarios de dichas municipalidades con los que fuimos conversando también nos contaron que cada taller era ocupado principalmente por mujeres que hacían parte de un mismo club.

Con estas informaciones en mano, nuestro segundo paso consistió en descartar aquellos talleres en los que pensamos que la conversación con las personas mayores se vería dificultada, al ser desafiada por el movimiento de los cuerpos, el ritmo de la música o de las ollas de cocina. En consecuencia, eliminamos de nuestra lista los talleres de cocina, gimnasia y baile.

Nuestro tercer paso fue seleccionar aquellos talleres en los que, según dedujimos, podría ser más fácil conversar. Nos quedamos con aquellos que nos parecían «más tranquilos»—otro ejemplo de nuestro desconocimiento inicial—, es decir, los que acogían actividades de tejido, bordado, pintura o manualidades. Estas acciones, pensamos, nos permitirían estar sentadas alrededor de una mesa y conversar con mayor facilidad con ellas (y no nos equivocamos). Derivado de estas primeras conjeturas, elegimos un taller de bordado (Providencia), un taller de tejido (Independencia) y un taller de cerámica (Santiago), construyendo diferentes estrategias de aproximación para cada cual.

La elección de un taller en cada una de las comunas seleccionadas marcó el inicio de nuestro trabajo de campo. Por lo general, los clubes de adultos mayores funcionan en los centros comunitarios que dependen de la municipalidad, o en otros espacios que las propias mujeres gestionan - juntas de vecinos y parroquias, entre otros-. Pero la organización de sus actividades ocurre de forma coparticipativa. Ellas predominan entre las participantes, ocupándose de la compra de equipos, materiales y también de la organización administrativa. Lejos de constituir una organización momentánea, espontánea o provisional, dichos clubes comunitarios son gestionados por entidades sin fines de lucro que fueron creadas por (y que agrupan a) vecinos y vecinas. Estos cuentan con personalidad jurídica, organigrama y estatuto propios, lo que les permite generar proyectos y obtener financiamientos junto con municipalidades, ministerios y 
otras subdivisiones administrativas del Estado. El carácter participativo y de gestión comunitaria feminizada de los clubes nos sorprendió desde las primeras incursiones en el terreno.

\section{La participación observante}

La práctica etnográfica de la participación observante fue llevada a cabo por el equipo de investigación de forma intensiva en cada taller durante un período extenso (al menos dieciséis meses en cada comuna, entre 2016 y 2018$)^{11}$, asistiendo a cada uno de ellos, puntualmente, cada semana ${ }^{12}$. Puntualizamos aquí que elegimos una forma muy particular de realizar esta etnografía: la participación observante. Esta apuesta metodológica invierte al método clásico antropológico, propuesto por Malinowski y conocido como observación participante. En este método clásico:

Los etnógrafos intentan ser participantes emocionalmente comprometidos y observadores fríamente desapasionados de las vidas de los otros. En la participación observante, los etnógrafos experimentan y observan su propia coparticipación y la de otros en el encuentro etnográfico. El cambio de una metodología a otra implica una transformación representacional en que, en lugar de elegir entre escribir una memoria etnográfica centrada en el Yo [self] o una monografía estándar centrada en el Otro, tanto el Yo como el Otro se presentan juntos en una narrativa etnográfica única, centrada en el carácter y proceso del diálogo etnográfico (Tedlock, 1991: 69. Traducción propia).

Al paso que nos fuimos inscribiendo a estos talleres como compañeras de actividades de las mujeres mayores, nos dimos cuenta de que cada taller era, en sí mismo, un club. Era a partir de las actividades recreativas de

11 Además de Gonzálvez (directora del proyecto), la etnografía contó con la participación intensiva de las asistentes Francisca Ortiz, Sofía Larrazábal, Alfonsina Ramírez y Catalina Cano. Asimismo, nos han asistido en momentos puntuales del desarrollo del trabajo Macarena Huaquimilla, Javiera Carrasco, Florencia Borquez y Natalia Arévalo. Guizardi solo se adentró en el proceso tras el fallecimiento de su madre, en el momento de análisis del material etnográfico (para triangular las informaciones y trabajar en la interpretación de los resultados).

12 Los talleres se llevaban a cabo los siguientes días y horarios: el taller de Providencia, los jueves por la tarde; el taller de Santiago, los martes en la mañana, y el taller de Independencia, el martes por la tarde. 
las clases que los clubes estructuraban todas o casi todas sus dinámicas de sociabilidad. Y esto era coincidente para las tres comunas de nuestra muestra. La única diferencia entre ellas es que en Santiago e Independencia los clubes ya estaban conformados por largo tiempo, mientras que, en Providencia, fuimos testigos del intento de creación de uno a partir de la sociabilidad que emergió en el taller. En todos ellos, la participación observante fue fundamental.

Durante el período del trabajo de campo, registramos, semana a semana, lo que sucedía en los talleres (conversaciones, experiencias, vivencias y emociones relacionadas con el envejecimiento y los cuidados), lo que dio origen a un conjunto de diarios de campo etnográficos. Estos registros etnográficos contenían información general de lo que sucede en el taller (historia de la conformación de los clubes, identificación de los roles de las integrantes, disposición del espacio, tipos de trabajos que realizar) e información referente a la vida y actividades diarias de las mujeres que lo conformaban (visiones sobre la vejez, vivencia sobre su propio envejecimiento, responsabilidades de cuidados, trabajo remunerado o no remunerado que desempeñan fuera y dentro de su hogar, temas de actualidad nacional e internacional y apreciaciones valóricas y sociales sobre salud, educación, política). A través de estas observaciones también fue posible realizar una caracterización de los grupos, recopilando datos sobre edad, estado civil, situación de pareja, parentesco y formas familiares, profesión u oficio, y situación laboral, de salud y de protección social de las participantes.

En total, el trabajo de campo en la comuna de Santiago contabilizó 54 observaciones y 21 relatos de vida de las mujeres mayores que los asistían, a partir de entrevistas en profundidad de una o dos sesiones cada una. En Providencia, se realizaron 40 observaciones y 12 relatos de vida. En Independencia se logró el registro etnográfico de 59 observaciones y 12 relatos de vida. Realizamos, además, 3 grupos focales de discusión ${ }^{13}$.

13 Siguiendo los requisitos éticos establecidos por la agencia financiadora del estudio, las personas que colaboraron tuvieron sus derechos explicados en un documento de consentimiento informado. Solo entrevistamos y registramos escenas de aquellas personas que aceptaron los términos de este documento. Asimismo, tanto los clubes como las mujeres fueron anonimizados: son citados en los artículos derivados de la investigación a través de pseudónimos (protegiéndose, así, sus identidades). Véanse Gonzálvez (2017) y Gonzálvez et alii (2019; 2020). 
Terminada la etapa de trabajo de campo, nos dedicamos al proceso de análisis de toda la información cualitativa (entre 2018 y 2019). Este análisis consistió en la construcción de una matriz de categorías emergentes que fueron estructuradas a través del software MaxQDA. Así, construimos un esquema conceptual de vinculación entre categorías y, además, de escalonamiento entre ellas, con la finalidad de contrastar las historias personales de las mujeres con las problemáticas específicas del cuidado en la vejez que ellas enfrentaban. Esta articulación tomó en consideración la asignación comunal de estas mujeres, permitiéndonos operacionalizar un cruce entre sus cursos de vida, el entorno comunal (en términos de estructuras públicas y políticas sociales destinadas a la vejez) y la posición de las mujeres en la estratificación social característica de cada comuna.

Con esto en mente, una vez construida esta matriz de categorías, analizamos todos y cada uno de los 153 relatos de campo etnográficos de las tres comunas, seleccionando escenas, citas, historias y pasajes de acuerdo con su pertinencia con cada una de estas categorías. Este material permitió una profunda inmersión en la relación entre cuidados comunitarios y mujeres mayores. Pero nos detendremos a continuación en aquello que constituye la antesala de este material empírico y su condición de existencia: la experiencia de acercamiento y negociación con las mujeres.

\section{Nuestro modo de relacionarnos}

La intención del diseño metodológico que utilizamos durante el transcurso de la investigación fue lograr una aproximación hacia las mujeres en la cual ellas se sintieran copartícipes de la investigación, conociendo lo que estábamos haciendo en cada una de las etapas de la etnografía. Para lograrlo, tuvimos muy presentes las palabras de Bárbara Macdonald cuando señalaba:

Los investigadores se acercan a las mujeres mayores que sirven a mujeres más jóvenes a lo largo de la vida y les piden servir una vez más, y luego cubren su vergüenza al irse diciendo sobre ellas que son como si fuesen sus abuelas, o madres, o tías. Y nadie, en la hermandad, las critica por eso (1989: 49). 
En primer lugar, durante toda la investigación, tomamos consciencia de que nuestras múltiples distinciones sociales (clase, edad, extranjería, entre otras) podían generar, al menos al inicio del trabajo de campo, ciertas distancias entre nosotras ${ }^{14}$. En sintonía con ello, y en un ejercicio de honestidad académica, durante el trabajo de campo nos preocupamos de ir realizando reflexiones con todo el equipo respecto de cómo percibíamos que estaba siendo nuestra relación con las mujeres mayores. Esto incluía conversar sobre cómo nos sentíamos en nuestra vinculación con ellas, sobre qué tipo de expectativas podíamos alentar con nuestra presencia, sobre cómo acoger los sentimientos que allí se expresaban, o cómo responder a sus muestras de afecto y cuidado hacia nosotras.

Pasados los meses, y a partir de nuestras reflexiones, pero, sobre todo, durante la escritura posterior de estas, nos dimos cuenta de que nuestra presencia en los clubes, semana tras semana, incluso como socias - como nos pasó, durante el segundo año, en la comuna de Independencia-, generó una vinculación particularmente situada y, por lo tanto, encarnada. Esto, nos invitó a reflexionar sobre la tríada de emociones de la que nos habla Jociles, cuando nos indica que el «trabajo de campo antropológico [...] es también el terreno de un triple juego: el que el etnógrafo juega con las emociones que experimenta, el que juega con la exteriorización de las mismas y, finalmente, el que juega con las emociones de los sujetos investigados» (2000: 33).

En este sentido, nuestra etnografía estuvo amparada en una perspectiva feminista desde abajo. Es decir, en una perspectiva atenta a la contradicción de la figura de autoridad de las investigadoras —reconociendo el lugar de subordinación que nosotras mismas ocupamos en el mundo académico debido a nuestra condición de género (Behar, 1995: 2) - y también a los matices políticos del proceso de constitución de las subjetividades entre

14 Con distinciones sociales, aludimos al debate de Bourdieu sobre las formas de diferenciación jugadas por los actores para establecer su ubicación en el campo social y su distancia en relación con los demás actores. Sabemos que estas estrategias no son neutrales - aun cuando se use un discurso naturalizador para justificarlas (2002: 67) 一, que constituyen un sentido estético (ibid.: 53), que involucran la incorporación de estructuraciones sociales de mediana y larga duración —los «condicionamientos de clase» (ibid.: 99)—, y que están, al mismo tiempo, vinculadas a la forma particular de cómo los sujetos asumen corporalmente - en cuanto habitus - sus capitales culturales, en la disputa por poder, posicionamiento y espacio social. En estas estrategias de distinción, las redes de contacto, relaciones y vínculos societarios a los que acceden o pueden acceder los sujetos. 
las personas estudiadas (Visweswaran, 2003: 74). Asumir estas jerarquías genéricas a las que estamos expuestas -investigadoras e investigadasconlleva reconocer que la tensión analítica entre particularismo y universalismo reproduce un ocultamiento de lo femenino como fuente de saber (Rosaldo, 1980). Por lo mismo, cualquier apuesta por una perspectiva feminista desde abajo debe estar comprometida con devolver el carácter parcial y situado del conocimiento científico, buscando con esto

restituir el valor del saber vivencial, implicado y comprometido. Un saber desde el que la razón y la emoción no sean dos polos distinguibles y contrapuestos como el pensamiento occidental androcéntrico y etnocéntrico nos quiere hacer creer, sobredimensionando el valor de lo «racional» frente a lo «emocional», lo científico frente a lo no científico y en el fondo lo masculino, en su asociación aristotélica con la razón, frente a lo femenino en su asociación con lo emocional (Gregorio y Alcázar, 2014: 2).

En nuestro caso, este proceso estuvo atravesado por nuestras subjetividades, militancias, saberes y genealogías, constituyendo, simultáneamente, nuestra propuesta de enfoque para los estudios de género y vejez apoyados en una perspectiva feminista. Nos parece fundamental expresar que absolutamente todo nuestro proceso etnográfico estuvo atravesado por el imperativo de las mujeres de negociar con nosotras los usos de nuestra metodología. Es decir, desde un primer momento, ellas dispusieron que negociarían incluso las formas de cómo las registraríamos. Esto, lejos de ser un detalle, configura un aspecto central para nuestro argumento en este artículo: la negociación de las asignaciones y distinciones es un procedimiento en el cual estas mujeres se sienten empoderadas. Hemos debido aprender con ellas las reglas de sus negociaciones para poder desarrollar el trabajo de campo. En este sentido, «negociar las distinciones» no era solamente una experiencia de las mujeres con su entorno social: era una práctica femenina de autocuidado a la que ellas nos han socializado. Negociar las distinciones es, por lo tanto, una praxis intersubjetiva en nuestra etnografía.

Al inicio del trabajo de campo, las sensaciones sobre esta negociación fueron de diverso tipo, involucrando las disposiciones espaciales de dónde podríamos o no situarnos en el espacio de los talleres. Predominaba 
en nosotras el nerviosismo, pero, sobre todo, la preocupación por si las mujeres mayores no estuvieran a gusto con nuestra permanencia allí. Esta inquietud no era infundada: ellas mismas nos decían al principio que no entendían bien por qué «teníamos que estar». Les respondíamos explicando nuestra idea de la etnografía.

Particularmente en el club de la comuna de Providencia, estas negociaciones metodológicas tuvieron una incré́ble impronta reflexiva. Las mujeres nos preguntaron, por ejemplo, si acaso no les haríamos encuestas, método de investigación social que les resultaba más familiar. Les llamó la atención cuando dijimos que no lo haríamos y, especialmente, cuando les explicamos que esto se debía a que preferíamos escucharlas y conocer cómo narraban sus historias (diario de campo, 18-8-2016). En una ocasión específica, doña Pilar nos preguntó detenidamente sobre los objetivos y preguntas de la investigación y nos entregó datos y perspectivas propias sobre el envejecimiento femenino en Chile (diario de campo, 29-9-2016).

Doña Pamela (68 años), a su vez, nos indagó sobre los tiempos de nuestra investigación, sobre cómo nos iba con el estudio de caso en otras comunas, si teníamos pautas de observación para guiar nuestra presencia en terreno. Concluyó, de nuestra conversación, que estábamos llevando a cabo aquello que «los antropólogos llaman observación participante» (diario de campo, 8-9-2016). Nos tendió, además, algunas consideraciones de su parte sobre formas de realizar las entrevistas en profundidad (diario de campo, 3 y 7-8-2017).

Cierto día, tras tres meses de convivencia con ellas, nos acercamos en un momento de descanso a algunas señoras para pedirles algunas informaciones personales. Doña Inés (91 años) quedó asombrada de que supiéramos tanto sobre ella: terminó de creernos cuando le dijimos que les prestábamos mucha atención a las cosas que nos contaban y que sus narraciones realmente nos interesaban (diario de campo, 29-9-2016). Semanas después, nos indagó si nos daba mucho trabajo anotar todo lo que ocurría: tenía curiosidad por saber cómo hacíamos los diarios (diario de campo, 24-11-2016). Moira (61 años) y Antonia (79 años) también manifestaron gran interés por conocer nuestros registros (diario de cam- 
po, 27-10-2016), con lo que les propusimos una dinámica: leerles algunas partes del diario de campo para que ellas pudieran conocerlo y opinar sobre él.

La propuesta fue acertada en muchos sentidos: permitió que ellas establecieran un contacto cercano con nuestro método de registro más «íntimo». En la primera de las lecturas que hicimos, ellas se concentraron enormemente. Algunas se reían e interaccionaban con lo que decíamos cuando sus nombres aparecían en la narración. Alejandra (74 años) comentó este día que era impresionante la cantidad de cosas que habíamos captado y registrado. En estas ocasiones, conversamos sobre la naturaleza sigilosa de varios de estos relatos, de nuestro cuidado en seleccionar aquello que se puede contar y en elegir formas éticas de contarlo. Estos ejercicios de compartir con ellas las narraciones terminaron generando un sentido de confidencia de ellas hacia nosotras, estableciendo lazos cercanos y de afecto (diario de campo, 29-12-2016).

Además, en todas las comunas explicitábamos que, si ellas no se sentían cómodas, que nos lo dijeran, pues nos marcharíamos: no queríamos, por nada del mundo, generar incomodidad. Con el paso del tiempo supimos que haber explicitado que era suya la última palabra fue muy importante para ellas. Nos contaron que habían tenido experiencias con profesionales que iban a hacerles encuestas o entrevistas y, luego, no volvían a saber nada más de ellos. Nos comprometimos con ellas a que esto no pasaría.

Debido a estas malas experiencias previas que ellas nos habían relatado, teníamos siempre en cuenta que la llegada de los/as etnógrafos/ as provoca emociones fuertes a la gente: ya sea porque se desplazan por primera vez hasta allí o porque se acercan a las personas desde la condición de investigadores/as (Jociles, 2000: 144-145). Con esto en mente, en el primer mes de etnografía, mantuvimos con las mujeres relaciones de sumo cuidado, con mucho respecto. Evitábamos, además, caer en un trato infantilizador (adoptado por algunos/as profesionales que trabajan con las personas mayores). Nos preocupaba que la diferencia de edad entre ellas y nosotras hiciera difícil construir una relación de proximidad. La edad y sus significados permearon nuestra entrada al terreno. Hasta 
que emergió una forma inicial de relacionarnos que nos permitió romper este hielo: modo nieta. Poco a poco, nuestro nerviosismo se fue transformando en cercanía, ternura, preocupación por ellas, y también de ellas hacia nosotras.

El modo nieta respondía al hecho de que nuestra experiencia más cercana con mujeres mayores la asociábamos, inicialmente, con nuestras propias relaciones de abuelidad. Aunque sabíamos que no todas las mujeres mayores eran abuelas, y mucho menos nuestras abuelas, este patrón relacional fue la primera vestidura que experimentamos. Aunque esta primera apuesta no ocurrió de la misma manera con todas las mujeres en todos los talleres, con el transcurrir de las semanas se amplió y, a la vez, se fue mutando. Muchas mujeres reivindicaban que no les gustaba que quienes no las conocían las situaran en la posición de abuelas. Por otro lado, algunas de ellas establecieron formas de relación con nosotras en las que la edad no significaba el elemento central (más que para compartir anécdotas o críticas). La heterogeneidad entre las mujeres de los talleres nos ofreció formas originales de relacionarnos con cada una de ellas. Más allá de la posición de parentesco que nuestra diferencia de edad podía proyectar, el reconocimiento como mujeres pareció vincularnos de manera más transversal: constituía un elemento de reconocimiento mutuo más interpelante para ellas que la cuestión generacional.

Con los cambios y el surgimiento de nuevos roles, Gonzálvez fue particularmente encajada por nuestras colaboradoras en el modo profesora. Ellas observaban que, en nuestro equipo, había diferentes posiciones de responsabilidad. La diferencia de edad entre González y las jóvenes asistentes de investigación no era tan grande (de unos diez años). No obstante, las mujeres de los talleres empezaron a denominarla «la profe». Cada vez que no estaba presente, las directoras de los clubes preguntaban a las jóvenes investigadoras: «¿cómo está la profe?»o, aun, «¡le pasó algo a la profe?». Tras saber que estaba todo bien, mandaban insistentes «saludos a la profe». Nos percatamos, entonces, de que los talleres estaban atravesados por relaciones jerárquicas que ellas querían que nosotras respetáramos. Por esto era tan importante para ellas identificar, reconocer y demarcar las jerarquías propias de nuestro equipo de investigación. 
Empezamos, así, a registrar que los talleres estaban compuestos por las alumnas, por una presidenta del club, una secretaria y una tesorera. Algunas mujeres ocupaban puestos de responsabilidad y el reconocimiento de su trabajo era una obligación recíproca en sus sistemas de intercambios de tareas y cuidados ${ }^{15}$.

Con el paso del tiempo, emergió también el modo militante. Lo adoptábamos en momentos en que personas externas a los talleres aparecían para ofrecer o pedir algo a las mujeres mayores. Personificamos este modo muchas veces durante las elecciones para los Gobiernos municipales de Chile, en 2016. Los candidatos a concejal de diferentes partidos políticos visitaban los clubes en búsqueda de votantes, anunciando qué harían por las mujeres mayores si fueran electos. En varias ocasiones, al terminar la presentación entregándonos el correspondiente regalo (calendarios, caramelos, una tarta para compartir), estos personajes se tomaban fotos sin pedir autorización de las mujeres.

Otra situación que nos empujaba al modo militante se producía con la visita, generalmente sin previo aviso, de personas que vendían las excursiones de fin de año, para ser financiadas con el presupuesto del Fondo de Desarrollo Vecinal (Fondeve) ${ }^{16}$, al que los clubes de mujeres postulaban. Los vendedores (generalmente hombres) ofrecían un pack turístico a algún destino cercano, incluyendo traslado, desayuno, comida, once ${ }^{17}$, baile, bingo y otras distracciones. Les hablaban siempre de sus «precios módicos». Nos llamaban la atención las frases cargadas de sexismo y machismo que usaban para convencer a las mujeres: «Podrían comer chancho [cerdo] hasta saciarse, sin que sus maridos se preocuparan por

15 Estas posiciones se explicitaban principalmente cuando las presidentas, en los diferentes clubes, se tomaban unos minutos de la clase — previo permiso de la profesora que impartía el taller- para tomar decisiones relacionadas con la excursión de fin de año, con la solicitud de alguna subvención a la municipalidad, o con recolectar dinero para apoyar a una socia en caso de necesidad.

16 El Fondo de Desarrollo Vecinal es un aporte que canalizan las municipalidades y al que acceden mediante concurso las juntas de vecinos, las organizaciones comunitarias funcionales (centros de madres, comités de seguridad y clubes del adultos mayores, entre otros) y organizaciones sin fines de lucro de cada comuna. Para acceder a este fondo, las organizaciones deben tener un año de constitución formal, no poseer deudas ni rendiciones pendientes con la municipalidad, y estar inscritas en el registro de personas jurídicas receptoras de fondos públicos.

$17 \mathrm{La}$ «once» refiere en Chile a la hora de la tarde en la que familias y amigos se reúnen para tomar una bebida caliente (té o café) con alimento dulce (trozo de pastel) y salado (pan con jamón o queso, por ejemplo). Sería un término equivalente a «la hora del té» o la «merienda». 
su aspecto». Otros incluso insinuaban que aparecerían «hombres con quienes realmente podrían pasarlo bien», dando a entender que las mujeres no tenían relaciones sexuales satisfactorias. En estas intervenciones, jamás les daban a las mujeres espacio para que opinaran sobre nada.

Frente a estas situaciones, asumimos abiertamente la postura mencionada por las perspectivas de las mujeres: nos juntábamos a ellas para frenar las fotos no autorizadas o, más divertido, hacerles bromas a estos visitantes, poniéndoles en ridículo por su falta de respeto. A los políticos, además, solíamos insistir en que dieran precisiones sobre sus propuestas. Por lo general, cuando se iban las visitas, las mujeres se sinceraban con nosotras sobre su molestia: «se creen que no nos damos cuenta de que lo que quieren es solo nuestro voto»; «que desagradable el señor que vino a vendernos la excursión, qué vulgar».

A partir de estos diversos modos de relacionarnos - nietas, profes, militantes - construimos nuestra presencia junto con ellas, adentrando a la reciprocidad de los cuidados que marca la experiencia colectiva de los clubes. Es decir, nuestra permanencia en los talleres por tantos meses implicó sostener un equilibrio consciente entre ellas y nosotras. Tejimos relaciones de cariño, y confianza, mientras compartíamos, semanalmente, conversaciones. En estos diálogos, reflexiones sobre sus historias y las nuestras comenzaron a entrelazarse. Configuramos, así, unos modos de relación por los que transitamos - no siempre con el mismo orden y forma- en las interacciones con cada una de las mujeres.

No obstante, estos modos de relacionarnos no se agotaron en los que aquí dejamos registrados, sino que, por el contrario, fueron tan heterogéneos como las mujeres con las que interactuamos, activándose unos $\mathrm{u}$ otros, dependiendo de quiénes fueran en ese momento parte de la relación. Estas formas de vincularnos a las que llamamos modos se convirtieron, consecuentemente, en nuestra «caja de herramientas analíticas», para parafrasear a Foucault $(1985)^{18}$. Predominó en nuestra etnografía una

18 Foucault (1985) considera que, en la investigación de procesos sociales, hay que crear categorías que sirvan como «una caja de herramientas». Esto implica construir instrumentos analíticos a partir una lógica propia a las relaciones de poder y a las luchas que se comprometen alrededor de ellas; una búsqueda que solo se puede hacer poco a poco, a partir de una reflexión (necesariamente histórica en algunas de sus dimensiones) sobre situaciones dadas. 
aproximación afectiva hacia las mujeres mayores que emergió genuinamente, y que permitió transcender las dicotomías yo-otro, sujeto-objeto (Caplan, 1993: 23).

Frecuentemente en las ciencias sociales, cuando una investigadora habla de sí misma como parte relacional de los campos que estudia, recibe la incómoda etiqueta de «subjetiva», una acusación de que su trabajo ya no es «científicamente bueno». Algunas autoras feministas denominan este procedimiento como «la mancha subjetiva» (Bell, 1993, citado en Gregorio, 2006). Este tratamiento acusatorio estigmatiza nuestras producciones como si se tratara de panfletos, como trabajo interesado y parcial. Todas estas etiquetas, propias del pensamiento racionalista de la ilustración, operan silenciando las lógicas relacionales femeninas y reproduciendo los dualismos patriarcales: parcial/imparcial, personal/ impersonal, emocional/racional (ibid.: 28-29).

Para nosotras, el tipo de relación que construimos es radicalmente intersubjetivo y no podría concebirse como neutral o políticamente desinteresado. En la interacción con las mujeres mayores, nos fuimos reconociendo las unas a las otras, buscando ir más allá del dualismo joven-vieja, extranjera-autóctona. Desde esta interacción, pudimos acceder a los saberes de nuestras colaboradoras, deconstruyendo muchos de los argumentos corrientes en Chile, según los cuales ellas son vistas como un «problema social». Esta forma de estar con ellas implicó dar un espacio a las emociones que sostuvieron nuestras relaciones. Nuestra forma de hacer trabajo de campo es - y fue-emocionalmente comprometida, situada, subjetiva y, por lo tanto, feminista.

\section{Conclusiones}

No es el lugar aquí para detenernos en toda la riqueza de detalles etnográficos que experimentamos en las incursiones etnográficas del proyecto cuyas delimitaciones metodológicas fueron abordadas en el presente artículo. Pero nos gustaría explicitar las principales inferencias teóricas a la que hemos llegado. Ellas devienen, en gran medida, del marco experiencial feminista que nos guio en términos metodológicos. 
El contacto sostenido semanalmente con las mujeres mayores en los clubes y sus actividades nos permitió profundizar en otros ejes de investigación. Dilucidamos las ambivalencias y contradicciones del envejecimiento femenino observando que, al paso que reproducen socialmente la vida, las mujeres son recordadas cotidianamente en el acto de cuidar (y con mucha más intensidad en la vejez) de los límites sociales, biológicos, culturales y políticos a que están expuestas, dada la prevalencia (hegemonía, diríamos) de las relaciones de poder patriarcales ${ }^{19}$. Conocer estos límites, que son a su vez reales y/o ficticios, impuestos y/o elegidos, y saber cómo las mujeres mayores conviven con ellos, pero también, cómo los resisten, y, sobre todo, cómo los subvierten a través de sus organizaciones comunitarias, fue uno de los cometidos de la investigación.

Todo lo que observamos y aprendimos con las protagonistas de nuestro estudio nos permite plantear que la sobrecarga productiva y reproductiva femenina y que las limitaciones y vulneraciones que ellas sufren distan muchísimo de ser la única dimensión de su vivencia de los cuidados. Nuestra etnografía abrió paso a la comprensión de que los cuidados son, contradictoria y simultáneamente, el eje articulador de diversas formas de enfrentar las vulneraciones e inequidades. A través del cuidado las mujeres mayores generan redes de ayuda mutua que constituyen herramientas sociales, políticas y económicas de resistencia femenina. Para ellas, cuidar a los demás en comunidad constituye un habilitador del cuidado de sí, y esta lógica constituye el corazón relacional de los clubes. Consecuentemente, estos serían espacios de aquello que Kunin denominó «prácticas de autocuidado paradójico»:

Podemos afirmar que el autocuidado paradójico implica, justamente, que «cuidarse» $\mathrm{y}$ «cuidar» a otros no son necesariamente prácticas ex-

19 Desde una perspectiva feminista crítica, el patriarcado constituye un fenómeno doble-dimensional. Por un lado, implica una dimensión «tópica», referente a la consecución de relaciones concretas, correspondiendo, así, al «nombre que recibe el orden de estatus en el caso del género», como «una estructura de relaciones entre posiciones jerárquicamente ordenadas que tiene consecuencias en el nivel observable» (Segato, 2010: 14). Pero, por otro lado, es también el estrato simbólico que ha conformado todas las formas de simbolismo. Las violencias de género y las sobrecargas productivas-reproductivas femeninas cumplen, en las sociedades patriarcales, una función central «en la reproducción de la economía simbólica del poder cuya marca es el género», constituyendo un acto necesario para la restauración de ese poder (ibid.: 13). 
cluyentes, como proponen las visiones esencialistas relativas a lo «femenino sacrificial» o a las visiones individualistas del feminismo blanco liberal. La solución para «tener tiempo para una» es tenerlo en simultáneo con tiempo «para otros». Es decir, haciendo convivir las tareas de cuidado de los hijos con las prácticas de autocuidado (2019:352).

Así, a través de las historias y experiencias compartidas con las mujeres de los clubes en la Región Metropolitana de Chile, observamos que el cuidado de sí era siempre el cuidado de las otras; que lo público y lo privado se extrapolaban y complementaban; que el afecto constituía parte del lenguaje político, a la vez que el cuidado constituía el centro de la organización de la vida económica de las mujeres (Gonzálvez et alii, 2019; 2020). Por todo ello, la etnografía nos condujo a asumir el cuidado como una experiencia dialéctica a partir de la cual las mujeres, al mismo tiempo en que sufren (y, frecuentemente, reproducen) las limitaciones y vulneraciones que la división patriarcal del trabajo sexual les imputa, también generan espacios de resistencia y ruptura (parcial, mayormente) de las desigualdades generizadas que marcan sus cursos de vida.

El cuidado también es, así, el articulador de redes femeninas, de sororidad, de visiones de mundo embebidas de las perspectivas propias de las mujeres. El cuidado es la forma en cómo ellas construyen y desafían (con toda la contradicción que esto implica) las fronteras del patriarcado. Con estas afirmaciones, nos situamos más allá de las perspectivas dicotómicas, bipolares, que suelen mirar al cuidado, bien como una cosa, bien como otra (Gonzálvez y Acosta, 2015). El cuidado es, precisamente, el campo de acción en el que se entreteje la relación dialéctica entre subalternización y agenciamiento femenino. Esta circunstancia demanda una mirada antropológica que, como decía Segato (2010), se hace desde una escucha prolongada y atenta a los matices y vicisitudes simbólicos de cada relación, experiencia y proceso.

Es en este campo de acciones entretejido alrededor de las múltiples facetas del cuidado que las mujeres disputan sus posiciones, negociando la forma en que pueden situarse en los intersticios de las imposiciones patriarcales. En este último sentido, el cuidado es, también, un territorio de batallas, un campo de conflictos. Y las mujeres actúan, precisamente, atrin- 
cherándose en redes femeninas de apoyo mutuo a partir de las cuales logran librar sus batallas - pequeñas, medianas o grandes- en contra de diversos elementos que reducen sus derechos, posibilidades y capacidades de acción. Al hacerlo, terminan construyendo su forma de habitar en el mundo. En este sentido específico, el habitar de las mujeres es un «habitar en el cuidado» desde la perspectiva que le da a este verbo Heidegger (1975: 150). Para el autor, habitar es una forma de construir espacios $y$ vinculaciones que solo puede existir mientras las personas se implican en ellos. Habitar es construir un espacio en que se habita de forma continua. Pero este construir como habitar «se despliega en el construir que cuida, a saber, el crecimiento, y en el construir que edifica construcciones [...]. Habitamos no porque hayamos construido, sino que construimos y hemos construido, en cuanto habitamos, esto es, en cuanto somos los habitantes» (ibid.: 153).

El punto central de nuestra reflexión invoca, entonces, a la consciencia antropológica de que no es posible desarrollar trabajo de campo etnográfico en un espacio con estas características sin integrar los sistemas de reciprocidad del cuidado - las obligaciones relacionales de dar, recibir y retribuir (Comas d'Argemir, 2017)— que los clubes comunitarios constituyen como su lógica más estructurante. Dicho de otra forma, sería inviable, e incluso violento, pretender observar estas lógicas recíprocas negándonos a participar de ellas. Así, la intersubjetividad no es una opción marginal, sino un imperativo empático: es necesaria como forma de reconocimiento de las redes de cuidado de las mujeres mayores en su forma de operar. Esto nos empujó, como explicitamos en este artículo, a desarrollar toda una serie de negociaciones con las mujeres, estableciendo los roles y modos de reflexividad que nuestra investigación asumiría. En síntesis, la investigación nos llevó a compartir con nuestras colaboradoras el lugar que ellas habitan en las trincheras del cuidado. Entendimos que sus vidas y sus historias son, en varios sentidos, un anuncio de experiencias que nos tocarán también a nosotras como mujeres que envejecen. Indagar sobre sus trayectorias y organizaciones comunitarias pasó a ser, para nosotras, un viaje al futuro. Uno en que ocupamos el lugar privilegiado de quienes pueden aprender de la experiencia de otras mujeres. 


\section{Bibliografía}

Barros, C., Fernández, B. y Herrera, S. (2014) «Nociones sobre la familia y las interrelaciones entre sus miembros de parte de los mayores de 45 años». Psicoperspectivas. Individuo y sociedad, 13 (1), 121-130.

Behar, R. (1995). «Introduction: Out of exile». En R. Behar y D. Gordon. Women writing culture. Berkeley: University of California Press.

BELL, D. (1993). «Yes Virginia, there is a feminist ethnography: reflections from three Australian fields». En D. Bell, P. Caplan y W. J. Karim. Gendered fields. Women, men and ethnography. New York: Routledge.

Bourdieu, P. (2002). La distinción. Criterios y bases sociales del gusto. Ciudad de México: Taurus.

CAPlan, P. (1993). «Introduction 2: The volume». En D. Bell, P. CAPlan y W. J. KaRIM. Gendered fields. Women, men and ethnography. New York: Routledge.

Comas D'Argemir, D. (2017). «El don y la reciprocidad tienen género: las bases morales de los cuidados». Quaderns-E, 22 (2), 17-32.

Comas D'Argemir, D. y Soronellas, M. (2019). «Men as carers in long-term caring: Doing gender and doing kinship». Journal of Family Issues, 40 (3), 315-339.

CEPAL (2007). Panorama social de América Latina y el Caribe. Santiago, Chile: Comisión Económica para América Latina y el Caribe (CEPAL).

De BeAuvorr, S. (1983[1970]). La vejez. Barcelona: Edhasa.

Del VAlLe, T. (2010). «La articulación del género y el parentesco desde la antropología feminista». En V. Fons, A. Piella, M. Valdés. Procreación, crianza y género. Aproximaciones antropológicas a la parentalidad. Barcelona: PPU.

Di LEONARdo, M. (1987). «The female world of cards and holidays: Women, families, and the work of kinship». Signs, 3 (12), 440-453.

ForTtes, P. (2016). La dependencia y apoyo a los cuidados, un asunto de derechos humanos. Santiago, Chile: Servicio Nacional del Adulto Mayor (SENAMA).

Foucault, M. (1985). Poderes y estrategias. Un diálogo sobre el poder y otras conversaciones. Madrid: Alianza. 
FreiXAs, A. (1997). «Envejecimiento y género: otras perspectivas necesarias». Anuario de Psicología , 73, 31-42.

Gallardo-Peralta, L., Sánchez-Moreno, E., Arias-Astray, A. y Barrón López DE Roda, A. (2015). «Elementos estructurales de la red social, fuentes de apoyo funcional, reciprocidad, apoyo comunitario y depresión en personas mayores en Chile». Anales de Psicología, 31, 1018-1029.

Gallardo-Peralta, L., Cuadra-Peralta, A., Cámara-Rojo, X., Gaspar-DelpiNo, B. y SÁnchez-Lillo, R. (2017). «Validación del inventario de envejecimiento exitoso en personas mayores chilenas». Revista Médica de Chile, 145 (2), 172-180.

Gibson, D. (1996). «Broken down by age and gender "the problem of old women" redefined». Gender and Society, 10 (4), 433-448.

GonzÁlvez, H. (2016). «El trabajo de parentesco en las familias en Santiago de Chile». Revista de Antropología Social, 25 (1), 153-169.

- (2017). «Ser mujer mayor en Santiago de Chile: feminización de los cuidados en la vejez y desigualdades acumuladas (Chile)». En A. VERA (ed.) Malestar socialy desigualdades en Chile. Santiago de Chile: Editorial Alberto Hurtado.

GonzÁlvez, H. y Acosta, E. (2015). «Cruzar las fronteras desde los cuidados: la migración transnacional más allá de las dicotomías analíticas». En M. GuizArdi (ed.) Las fronteras del transnacionalismo. Límites y desbordes de la experiencia migrante en el centro y norte de Chile. Santiago de Chile: Ocho Libros.

Gonzálvez, H., Guizardi, M., Ramírez, A. y Cano, C. (2019). «El club como trinchera. Una etnografía sobre cuidados comunitarios entre mujeres mayores en Independencia (Chile)». Revista de Antropología Social, 28, 317-348.

GonZÁlvez, H. y Guizardi, M. (2020). «Las mujeres y el envejecimiento en la investigación social (1950-2018)». Revista Estudos Feministas, 28 (1), 1-14.

GonzÁlvez, H. y LarrazÁbal, S. (2019). «Familias y religiosidad en Santiago de Chile: nuevos significados de las prácticas religiosas». Perfiles Latinoamericanos, 27 (54), 1-23. 
Gonzálvez, H., Larrazabal, S. y Guizardi, M. (2020). «Negociar las distinciones. Una etnografía sobre género y cuidados en un taller de bordados para señoras mayores en Providencia (Chile)». Chungará (Arica), 52 (1), 143-159.

Gonzálvez, H., Ortiz, F. y Espinoza, A. (2018). «Redes de parentesco en las familias en Santiago de Chile». Revista Hispana para el análisis de Redes Sociales, 29 (1), 111-124.

Gregorio, C. (2006). «Contribuciones feministas a problemas epistemológicos de la disciplina antropológica: representación y relaciones de poder». AIBR. Revista de Antropología Iberoamericana, I (1), 22-39.

- (2011). «Análisis de las migraciones transnacionales en el contexto español, revisitando la categoría de género desde una perspectiva etnográfica y feminista». Nueva Antropología, 14 (74), 39-72.

Gregorio, C. y AlcÁZAr, A. (2014). «Trabajo de campo en contextos racializados y sexualizados. Cuando la decolonialidad se inscribe en nuestros cuerpos (España)». Gazeta de Antropología, 30 (3), 1-16.

Gregorio, C. y Gonzálvez, H. (2012). «Las articulaciones de género y parentesco en el contexto migratorio: Más allá de la maternidad transnacional». Ankulegui, 16, 43-57.

Guajardo, G., Tijoux, M. E. y Abusleme, M. T. (eds.) (2015). La construcción social de las demencias en las personas mayores de la Región Metropolitana, Chile. Santiago, Chile: SENAMA, FLACSO Chile, Instituto Chileno de Terapia Familiar.

Heidegger, M. (1975). «Construir, habitar, pensar». Teoría , (5-6), 150-163.

Herrera, M. (2011). Predictores de un buen envejecer. Chile y sus mayores. Resultados tercera encuesta nacional calidad de vida en la vejez. Santiago de Chile: Pontificia Universidad Católica de Chile.

Huenchuan, S. (2014). "¿Qué más puedo esperar a mi edad? Cuidado, derechos de las personas mayores y obligaciones del Estado (Chile)». Autonomía y dignidad en la vejez: teoría y práctica en políticas de derechos de las personas mayores. Santiago de Chile: Comisión Económica para América Latina.

Instituto Nacional de Estadísticas (INE) (2017). Resultado de población por sexo y edad (Chile). Santiago: Instituto Nacional de Estadísticas. 
Jociles, M. (2000). «Trabajo de campo, emociones e interpretación». En C. Lisón-Tolosana (ed.). Antropología: Horizontes interpretativos. Granada: Universidad de Granada.

Kunin, J. (2019). El poder del cuidado: Mujeres y agencia en la pampa sojera argentina. Tesis de doctorado. Programa de Posgrado en Antropología Social y Cultural. Instituto de Altos Estudios Sociales, Universidad Nacional de San Martín. Buenos Aires, Argentina.

Macdonald, B. (1989). "Outside the sisterhood: Ageism in women's studies». Women's Studies Quarterly, 17 (1/2), 6-11.

Mazzucchelli, N. y ArÉvalo, A. (2019). «Personas mayores y sexualidad. Relatos y experiencias del taller "Con la sexualidad nunca se baja el telón”». Revista Pensamiento y Acción Interdisciplinaria, 5 (1), 8-28.

Mazzucchelli, N. (2019). «Envejecimiento positivo para Chile: ¿Una vejez sin Estado?» Revista Kairós-Gerontologia, 22 (3), 25-42.

Ministerio de Desarrollo Social (2013). Encuesta de caracterización socioeconómica nacional 2013. Una medición de la pobreza moderna y transparente para Chile. Santiago, Chile: Ministerio de Desarrollo Social.

- (2015a). Encuesta de caracterización socioeconómica nacional 2015. Ampliando la mirada sobre la pobreza y la desigualdad. Santiago, Chile: Ministerio de Desarrollo Social.

- (2015b). Documento pobreza y distribución de ingresos Región Metropolitana de Santiago: Resultados Encuesta Casen 2015. Santiago, Chile: Ministerio de Desarrollo Social.

Ortiz, F. y GonzÁlvez, H. (2017). «Brechas y contradicciones entre la familia definida por la Ley y las familias en Santiago de Chile: miradas desde la antropología a partir de la dicotomía biología versus elección». RUMBOS TS. Un Espacio Crítico para la Reflexión en Ciencias Sociales, $17,37-56$.

Osorio, P. (2006). «La longevidad: más allá de la biología. Aspectos socioculturales». Papeles del CEIC, 22 , 1-28. (2007). «Construcción Social de la Vejez y Expectativas ante la Jubilación en Mujeres Chilenas». Universum (Talca), 22 (2), 194-212. 
Rodríguez, C. (2015). «Economía feminista y economía del cuidado. Aportes conceptuales para el estudio de la desigualdad». Nueva Sociedad, 256, 30-44.

Rosaldo, M. (1980). «The use and abuse of anthropology: reflections on feminism and cross-cultural understanding». Signs: Journal of Women in Culture and Society, 5 (3), 389-417.

SEGATO, R. L. (2010). Las estructuras elementales de la violencia. Ensayos sobre género entre la antropología, psicoanálisis y los derechos humanos. Buenos Aires: Prometeo.

Sojo, A. (2011). «De la evanescencia a la mira: El cuidado como eje de políticas y de actores en América Latina». CEPAL, Serie Seminarios y Conferencias, (67), 1-70.

TedLock, B. (1991). «From participant observation to the observation of participation: The emergence of narrative ethnography». Journal of Anthropological Research, 47 (1), 69-94.

Visweswaran, K. (2003). Defining feminist ethnography. Turning points in qualitative research: Tying knots in a handkerchief. New York: Altamira Press. 